\title{
ORIGINAL
}

\section{SEGUIMIENTO DE 1991 A 2004 DE LA MORTALIDAD Y LOS FACTORES DE RIESGO EMERGENTES EN UNA COHORTE DE POBLACIÓN GENERAL ESPAÑOLA. ESTUDIO DRECE III (DIETA Y RIESGO DE ENFERMEDADES CARDIOVASCULARES EN ESPAÑA) (*)}

\author{
Agustín Gómez de la Cámara (1) Miguel Angel Rubio Herrera (2) José Antonio Gutiérrez Fuentes \\ (3) Juan Antonio Gómez Gerique (4) Jorge del Campo (3) César Jurado Valenzuela (1) José \\ Lapetra Peralta (5) Elena Melús Palazón (6) y Nieves Rodríguez Pascual (1) \\ (1) Hospital Universitario 12 de Octubre. Unidad de Investigación. CIBER de Epidemiología y Salud Pública. \\ Madrid. \\ (2) Servicio de Endocrinología y Nutrición. Hospital Clínico Universitario de San Carlos. Madrid. \\ (3) Instituto DRECE de Estudios Biomédicos. Madrid. \\ (4) Servicio de Bioquímica Clínica. Hospital Marqués de Valdecilla. Santander. \\ (5) Unidad Docente de Medicina Familiar y Comunitaria. Sevilla. \\ (6) Centro de Salud Actur Sur. Zaragoza. \\ En representación del grupo DRECE: Anexo.
}

\section{RESUMEN}

Fundamento: El estudio DRECE III se basa en el seguimiento de una cohorte de población general representativa de la sociedad española. El objetivo de este trabajo es analizar, tras 13 años de seguimiento, la mortalidad general, la distribución de la mortalidad por causas y los principales factores de riesgo asociados.

Métodos: La cohorte DRECE se compone de 4.783 sujetos seguidos desde 1991 hasta 2004, el rango de edad al final se extiende desde los 18 a 73 años. Las personas participantes fueron seleccionadas mediante muestreo estratificado polietápico. Las causas de mortalidad han sido proporcionados por el Instituto nacional de Estadística. Las tasas se calcularon mediante regresión de Poisson y la identificación de factores de riesgo mediante la regresión de riesgos proporcionales de Cox.

Resultados: En el periodo 1991-2004 fallecieron 125 sujetos, lo que se corresponde con una tasa de mortalidad total de 1.76 por 1000 habitantes. La distribución por causa de muerte fue: 53 personas por cáncer $(42,4 \%)$ y 31 por causas circulatorias $(24,8 \%$ ) (24 por causa cardiovascular). Las 41 defunciones restantes $(32,8 \%)$ se englobaron dentro de la categoría CIE 10 de "Otras". Para la mortalidad total las variables independientes asociadas fueron: creatinina>1,5 mgr/dl, hazard ratio (HR) 3.78(IC 95\%: 1,52-9,40); diabetes, HR 2,80; sexo masculino, HR 2,39 (I C95\%: 1,61-3,55) ; edad, HR 1,08 (IC95\%: 1,07-1,10); gammaglutamil transpeptidasa, HR 1,001 (IC95\%: 1,0001,003). Factores de riesgo asociados a cáncer fueron: edad, HR 1,12 (IC95\%: 1,09-1,16); y tabaco, HR 1,33 (IC 95\%: 1,14-1,54). Para la mortalidad cardiovascular fueron: creatinina $>1.5 \mathrm{mg} / \mathrm{dl}$, HR 19,40 (IC 95\%: 5,45-69,12); diabetes, HR 9,82 (IC 95\%: 4,19-23,04); y edad, HR 1,10 (IC 95\%: 1,05-1,15).

Conclusión: Se obtuvo un patrón de mortalidad en el que predomina el cáncer como causa más frecuente. Aparecen factores de riesgo clásicos asociados a las principales causas. La diabetes emerge como un factor muy llamativo asociado a mortalidad precoz. La dieta no se asoció a la mortalidad total.

Palabras clave: Mortalidad. Enfermedades cardiovasculares. Cáncer. Factores de riesgo. Estudio de cohortes. España.

\section{Correspondencia:}

Agustín Gómez de la Cámara

Unidad de Investigación-Epidemiología Clínica.

Hospital Universitario 12 de Octubre.

CIBER de Epidemiología y Salud Pública

acamara@h12o.es

\section{ABSTRACT}

\section{1-2004 Follow up of an Spanish}

General Population Cohort. Mortality and Raising Risk Factors in the DRECE III Study.( Diet and Risk of

\section{Cardiovascular Diseases in Spain)}

Background: The DRECE III study is based on the follow up of a cohort representative of the Spanish general population. The mortality, its main causes and relevant risk factors have been analyzed.

Methods: The DRECE cohort is composed of 4783 subjects followed since 1991 to 2004 (70930 person-years). In 1991 a general medical exam including blood analysis and nutritional questionnaire was undertaken. Currently the age spam is from 18 to 73 years. Vital status and mortality causes were provided by the National Institute of Statistics.

Results: In this period, 125 deaths were registered: 53 persons $(42.4 \%)$ due to cancer; 31 persons ( $24.8 \%$ ) due to circulatory causes, of which 24 were due to cardiovascular origin. The remaining $41(32.8 \%)$ deaths were included under the ICD 10 "Other chapters". For the all causes mortality the independent associated variables were: creatinina $>1.5 \mathrm{mgr} / \mathrm{dl}$, HR 3.78 (95\% CI: 1.52 9.40); diabetes, HR 2.80 (95\% CI: 1.74-4.46); male sex, HR 2.39 (I95\% CI: 1.61-3.55); age, HR 1.08 (I95\% CI: 1.07-1.10); and gammaglutamil transpeptidasa, HR 1.001 (195\% CI: 1.000-1.003). In the case of cancer mortality the risk factors founded were: age, HR 1.12 (I95\% CI: 1.09-1.16); and tobacco, HR 1.33 (I95\% CI: 1.14-1.54). For cardiovascular mortality were creatinina $>1.5 \mathrm{mg} / \mathrm{dl}$, HR 19.40 (I95\% CI: 5.45-69.12); diabetes, HR 9.82 (I95\% CI: 4.19-23.04); and age, HR 1.10 (I95\% CI: 1.05-1.15).

Conclusions: We obtain a mortality pattern where cancer is the most frequent cause. Classic risk factors appear associated to main death causes. Diabetes mellitus has an outstanding role as risk factor associated to early mortality. No specific diet data was associated neither to all causes mortality, nor to cardiovascular or cancer.

Key words: Mortality. Cardiovascular diseases. Cancer. Risk factors.

(*) Estudio financiado en parte por ayuda FIS 03/0014 y Grupo SOS. 


\section{INTRODUCCIÓN}

El Proyecto DRECE fue diseñado para conocer la prevalencia de factores de riesgo cardiovasculares en la población española y su relación con los hábitos dietéticos. El estudio se inició con un corte trasversal en 1991 (DRECE I) ${ }^{1-3}$ y la creación de un registro conteniendo datos demográficos, clínicos, factores mayores de riesgo cardiovascular, perfil de laboratorio (perfil lipídico y otras determinaciones bioquímicas básicas), y las respuestas de una encuesta dietética homologada. Los principales resultados de este estudio mostraron que la prevalencia de factores de riesgo no mostraba diferencias relevantes con la encontrada en otras sociedades occidentales ${ }^{4-7}$. Sin embargo, los datos de mortalidad en España parecían indicar la existencia de una clara paradoja entre ambas realidades: una mortalidad baja y la prevalencia de factores de riesgo relativamente elevada. Cinco años después se abordó sobre un subgrupo de la cohorte inicial el estudio DRECE II $^{8,9}$ que permitió demostrar, a pesar del escaso tiempo transcurrido, las diferencias de morbilidad entre quienes en 1991 presentaban un patrón de riesgo para enfermedades cardiovasculares (ECV) y aquellos que no lo presentaban (probabilidad de desarrollar algún tipo de accidente coronario 3,9 veces superior). El estudio también puso de manifiesto cambios y tendencias desfavorables del perfil lipídico y de los patrones alimentarios, siendo este empeoramiento significativamente superior en las zonas más industrializadas. Transcurridos más de 12 años de seguimiento se inicició el estudio DRECE III que, como uno de sus objetivos principales, pretende establecer las relaciones entre las características epidemiológicas, clínicas y hábitos alimentarios iniciales de la cohorte DRECE y la mortalidad total, así como su distribución por sus principales causas. Incluye también la observación de la evolución y tendencia del patrón alimentario, perfil de factores de riesgo, y perfil lipídico en la población.

\section{SUJETOS Y MÉTODOS}

Diseño del estudio: Se trata de un estudio observacional y descriptivo tipo cohorte histórica. Ámbito del estudio: población general representativa de distintos puntos de la geografía española participantes en el estudio DRECE I cuya selección y muestreo se han descrito previamente ${ }^{1,2}$.Sujetos de estudio: La cohorte DRECE está formada por 4.783 sujetos cuya evolución se ha seguido desde 1991 hasta 2004, y a esta fecha tienen unas edades comprendidas entre 18 y 73 años al final del seguimiento. Su estatus vital y causa de mortalidad han sido proporcionados por el Instituto Nacional de Estadística mediante convenio. Procedimientos: a todos los integrantes de la cohorte DRECE en 1991 se les realizó una exploración médica, anamnesis familiar y personal con cuestionario nutricional y sobre actividad física, así como pruebas complementarias de laboratorio ${ }^{1,2}$.

Variables y mediciones: Datos antropométricos y demográficos: edad, sexo, talla, peso -IMC- y perímetros. Datos clínicos y epidemiológicos: exploración física básica (incluyendo presión arterial y frecuencia cardiaca), perfil lipídico (colesterol total, triglicéridos totales, cHDL, cLDL, VLDL, apo A-I y apo B), perfil bioquímico básico (glucosa, urea, ácido úrico, creatinina, $\mathrm{Ca}, \mathrm{Mg}$, proteínas totales, albúmina, GOT, GGT y GPT). Datos nutricionales: la encuesta sobre hábitos alimentarios se realizó mediante un cuestionario de frecuencia de consumo de alimentos (CFCA) desarrollado y validado para la población adulta española ${ }^{10}$. Datos de mortalidad: aportados por el Instituto Nacional de Estadística (INE), por todas las causas, en base a los certifi- 
cados de defunción registrados. Plan de análisis: La información obtenida fue depositada en un soporte informático mediante procedimientos estandarizados y sucesivos controles de calidad (reintroducción, rangos lógicos). La explotación estadística comenzó por un análisis descriptivo. Las variables cualitativas se describieron mediante frecuencias absolutas y relativas, las variables cuantitativas con distribución normal se resumieron mediante su media, desviación estándar, valor mínimo, máximo, mientras que las variables claramente no normales se resumieron mediante su mediana y cuartiles. Algunas variables de naturaleza continua se recodificaron a categóricas como el colesterol total, cHDL, cLDL, creatinina según puntos de corte tradicionales para obtener una valoración del riesgo clínicamente más operativa. Variables de varias categorías como la consideración de antecedentes familiares se convirtieron en dicotómicas según la presencia o no del antecedente de interés (diabetes, infarto de miocardio, aterosclerosis...etc.). El análisis descriptivo se realizó además de manera estratificada por sexo y estratos de edad y se hicieron comparaciones mediante análisis de la variancia (ANOVA) y test Ji-cuadrado de acuerdo a la naturaleza de la variable. Las tasas de mortalidad se estimaron mediante regresión de Poisson con ajustes por edad, sexo y años de seguimiento. Para valorar la relación entre los distintos factores o características y la supervivencia de los sujetos de estudio se utilizó la técnica de regresión de riesgos proporcionales de Cox. Las premisas de riesgo proporcional de la regresión de Cox se verificaron mediante el Test Supremun. Para su desarrollo se utilizó el enunciado ASSESS en al procedimiento PROC PHREG del paquete estadístico SAS. Se consideró un valor $p$ de $<0,05$ para verificar la significación estadística. Se realizó un primer estudio de asociación bivariable entre los factores de riesgo (basales) tradicionales para constatar que se trataban de factores de riesgo en nuestra muestra. Los estimadores de riesgo (hazard ratio) asociados se calcularon para cada uno y se presentaron junto con sus intervalos de confianza al $95 \%$.

Se consideró la supervivencia total y la supervivencia cardiovascular y por cáncer por separado. En una segunda fase para la búsqueda de un modelo final, y dada la cantidad de factores considerados, se completó el estudio con un análisis multivariable, considerando tanto aquellos factores de riesgo con resultado significativo en el análisis bivariable (o una significación para la chi ${ }^{2}$ del estimador $\leq 0,20$ ), como aquéllos que tenían cierto interés o plausibilidad dentro de los objetivos del estudio.

\section{RESULTADOS}

De un total de 4.783 individuos que formaban la cohorte DRECE, entre 1991 y 2004 fallecieron 125 sujetos, 89 fueron varones y 36 fueron mujeres, según datos aportados por el INE. La distribución por causa de muerte fue la siguiente: 53 personas por cáncer $(42,4 \%) ; 31$ personas por causas circulatorias $(24,8 \%)$, de los cuales 24 se debieron a causas cardiovasculares; y los 41 restantes $(32,8 \%)$ se englobaron dentro de la categoría de "Otras". Las principales causas específicas se muestran en la tabla 1.

La tasa de mortalidad ha sido de 1,76 por 1000 habitantes/año (70.930 personasaños). Por sexo 89 fallecidos eran hombres y 36 mujeres, con tasas de 2,57 y 0,99 por mil habitantes/año respectivamente (34579 y 36351 personas-año respectivamente). La edad media de la cohorte al inicio y al final del estudio fue de 30,48 años y 46,28 años respectivamente. La edad media en cada grupo de causas de mortalidad fue de 
Tabla 1

Principales causas de mortalidad específicas

\begin{tabular}{|c|c|c|}
\hline Causa de muerte CIE 10 & Causa de fallecimiento & Defunciones \\
\hline C349 & Neoplasia maligna de bronquio y pulmón, no especificada & 11 \\
\hline I 219 & Infarto agudo del miocardio, sin otra especificación & 9 \\
\hline $\mathrm{I} 259$ & Enfermedad isquémica crónica del corazón, no especificada & 7 \\
\hline C509 & Tumor maligno de la mama, parte no especificada & 5 \\
\hline C189 & Tumor maligno del colon, parte no especificada & 5 \\
\hline $\mathrm{C} 259$ & Tumor maligno del páncreas, parte no especificada & 5 \\
\hline $\mathrm{C} 169$ & Tumor maligno del estómago, parte no especificada & 4 \\
\hline K746 & Otras ciHRosis del hígado y las no especificadas & 4 \\
\hline 1991 & Otras neoplasias malignas de localización no especificada & 2 \\
\hline C859 & Linfoma no Hodgkin, no especificado & 2 \\
\hline 91(CIE-9) & Colitis, enteritis y gastroenteritis de supuesto origen infeccioso & 2 \\
\hline 340(CIE-9) & Esclerosis múltiple & 2 \\
\hline 4254(CIE-9) & Otras cardiomiopatías primarias & 2 \\
\hline 4275(CIE-9) & Parada cardiaca & 2 \\
\hline 9289(CIE-9) & Lesión por aplastamiento de una zona no especificada del miembro inferior & 2 \\
\hline J449 & Enfermedad pulmonar obstructiva crónica, no especificada & 2 \\
\hline $\mathrm{C} 20$ & Tumor maligno del recto & 1 \\
\hline
\end{tabular}

Tabla 2

Principales factores de riesgo asociados Análisis univariante

\begin{tabular}{|c|c|c|}
\hline Variable & valor $\mathbf{P}$ & $\begin{array}{c}\text { Hazard Ratio } \\
\text { (intervalos de confianza 95\%) }\end{array}$ \\
\hline Antecedentes personales de IAM & $<0,0001$ & $29,06(15,62-54,09)$ \\
\hline Antecedentes personales de angor & $<0,0001$ & $11,91(6,40-22,13)$ \\
\hline Creatinina $(>1,5 \mathrm{mg} / \mathrm{dl}) *$ & $<0,0001$ & $10,99(4,49-26,89)$ \\
\hline creatinina $(\mathrm{mg} / \mathrm{dl}) * *$ & $<0,0001$ & $8,59(4,89-15,10)$ \\
\hline diabetes mellitus(>126 mg/dl) & $<0,0001$ & $7,61(4,84-11,97)$ \\
\hline Aterosclerosis & $<0,0001$ & $7,06(4,16-11,99)$ \\
\hline Antecedente personal DM & $<0,0001$ & $6,76(4,25-10,76)$ \\
\hline Antecedentes familiares cardiopatía & $<0,0001$ & $5,05(2,64-9,67)$ \\
\hline HTA-OMS & $<0,0001$ & $4,14(2,88-5,93)$ \\
\hline HTA - JNC VI & $<0,0001$ & $3,18(1,97-5,14)$ \\
\hline Antecedentes personales de HTA & $<0,0001$ & $3,09(1,84-5,20)$ \\
\hline Colesterol total $(>200 \mathrm{mg} / \mathrm{dl})$ & $<0,0001$ & $3,07(2,13-4,43)$ \\
\hline Creatinina $(>1,2 \mathrm{mg} / \mathrm{dl})$ & $<0,0001$ & $2,80(1,74-4,53)$ \\
\hline Colesterol total $(>240 \mathrm{mg} / \mathrm{dl})$ & $<0,0001$ & $2,58(1,75-3,80)$ \\
\hline cLDL (>120 mg/dl) & $<0,0001$ & $2,63(1,82-3,79)$ \\
\hline cLDL (>160 mg/dl) & $<0,0001$ & $2,37(1,60-3,51)$ \\
\hline
\end{tabular}

IAM: infarto agudo de miocardio; ECV: enfermedad cardiovascular; HTA: hipertensión arterial; cLDL: colesterol unido a lipoproteínas de baja densidad; cHDL: colesterol unido a lipoproteínas de alta densidad; HTA criterio OMS y HTA criterio JNC VI *Variable dicotómica **variable continua.

56,62 años para la mortalidad por todas las causas; de 58,32 años para cáncer; de 59,45 años para circulatorias-cardiovasculares y de 52,29 años para el epígrafe de "Otras" causas. Los factores de riesgo aso- ciados a la mortalidad total, abordaje univariante, se muestran en la tabla 2. Predominan los factores de riesgo cardiovascular. Los factores de riesgo más fuertemente asociados son los antecedentes personales 
Tabla 3

Factores de riesgo de mortalidad Total datos ajustados

\begin{tabular}{|l|c|}
\hline \multicolumn{2}{|c|}{ Análisis de riesgos proporcionales de Cox } \\
\hline \multicolumn{1}{|c|}{ Variable } & hazard ratio (IC95\%) \\
\hline Diabetes mellitas (>126 mg/dl) & $2,806(1,764-4,465)$ \\
\hline Sexo (varón) & $2,399(1,615-3,558)$ \\
\hline Edad & $1,087(1,070-1,105)$ \\
\hline Creatinina $>15 \mathrm{mg} / \mathrm{dl}$ & $3,781(1,520-9,408)$ \\
\hline GGT & $1,001(1,000-1,003)$ \\
\hline
\end{tabular}

GGT: gamma-glutamil-transpeptidasa.

y familiares de infarto agudo de miocardio y de angor. Los factores independientes después del ajuste multivariable se muestran en la tabla 3. Debemos hacer notar que no se han introducido en los análisis de ajuste finales los antecedentes personales o familiares, ya que acaparaban los modelos predictivos ocultando información de interés. Para la mortalidad específica por cáncer los factores son la edad, HR 1,12 (IC 95\%: 1,09-1,16); y el tabaco, HR 1,33 (IC 95\%: 1,14-1,54). Para la mortalidad cardiovascular lo son la creatinina $>1,5 \mathrm{mg} / \mathrm{dl}$, HR 19,40 (IC 95\%: 5,4569,12); la diabetes, HR 9,82 (IC 95\%:4,19-23,04); y la edad, HR 1.10 (IC 95\%:1,05-1,15).

\section{DISCUSIÓN}

La tasa de mortalidad de la cohorte en el periodo 1991-2004 fue de 1,76 por 1000 habitantes, similar a las tasas de mortalidad en España para los mismos años de seguimiento, rango de edad y sexo, siendo muy inferiores a las observadas en el resto de países europeos ${ }^{11}$. Obviamente distan de las tasas de mortalidad total de la población española, que en 1991 y 2003 respectivamente fueron de 8,67 y 9,16 por 1000 habitantes. Ello es debido a que el rango de edad de nuestra cohorte al inicio del estudio es 5 a 60 años y no recoge el grueso de mortalidad que en España se produce en los grupos de edad de 65 a 74 y de $>75$ años ${ }^{12,13}$.
Para estos grupos de 5 a 60 años durante estos doce años de seguimiento el cáncer fue la principal causa de muerte. Debe llamarse la atención sobre la identidad de los cánceres más frecuentes: carcinoma broncogénico y de pulmón, mama, y colon. Aún así, hay que destacar que el infarto de miocardio es la segunda causa específica de muerte. Esta circunstancia ya ha sido recogida en estudios previos ${ }^{14}$ y se ratifica en nuestro estudio. De este patrón de mortalidad deben deducirse importantes implicaciones no sólo en términos de salud pública sino respecto a la repercusión que este perfil de mortalidad supone en la actividad asistencial sanitaria diaria.

Las ECV han sido consideradas tradicionalmente como la primera causa de muerte en los países desarrollados para la población general ${ }^{1}$ pero si nos hubiéramos extendido hasta mayores edades quizás ya no hubiéramos observado lo mismo en este estudio. El informe de la Sociedad Española de Arteriosclerosis de 2003 sobre la mortalidad en la sociedad española ${ }^{15}$, señala que la tasa de mortalidad cardiovascular aumenta más intensamente a medida que se incrementa la edad, siendo superior a mil por 100.000 habitantes en las personas mayores de 70 años, y provocando que para el conjunto de la población las enfermedades del aparato circulatorio ocupen todavía el primer lugar como causa de muerte, ya que los ancianos son los que tienen tasas de mortalidad más elevadas y por tanto contribuyen en mayor medida a conformar el patrón de mortalidad total general. No obstante se observa en estudios recientes que esta situación ha ido cambiando, siendo en la actualidad el cáncer la primera causa de muerte en algunos países ${ }^{16,17}$. Probablemente en España vaya en esa dirección también. La evolución en la mortalidad proporcional por cáncer y enfermedades cardiovasculares, (no por enfermedades del aparato circu- 
latorio) en la población española (INE) pasa de una ratio cáncer/cardiovascular de 0,99 en 1991 a 1,25 en 2003. La Sociedad Americana del Cáncer ${ }^{15}$ ha observado que cuando se agregan los datos de mortalidad por edad, el cáncer supera a la enfermedad cardiaca como la principal causa de muerte en personas menores de 85 años desde 1999, siendo el cáncer de pulmón y bronquios el más frecuente, al igual que ocurre en nuestra cohorte. Por otra parte, en un estudio europeo sobre una cohorte de una población griega con características similares a la población española, en el que participaron 28.572 personas, de entre 20 y 86 años, con un seguimiento de 3,7 años, se observaron 275 fallecimientos, 54 (19\%) de ellos por enfermedad coronaria y 97 $(35 \%)$ por cáncer ${ }^{17}$, resultados que coinciden con nuestros hallazgos. En el estudio citado Se observó también una asociación clara entre la dieta mediterránea estricta y la reducción de mortalidad total que ya había sido advertida por otros estudios recientes. ${ }^{18}$

Los factores de riesgos asociados a la mortalidad general que emergen en el análisis univariante son prácticamente aquellos reconocidos como factores de riesgo cardiovascular clásicos para distintos puntos de corte o clasificaciones. El estudio estaba diseñado originalmente para tal fin y quedan bien expresados, dominando el cuadro de riesgo general. Por otro lado son poco conocidos los factores de riesgo del cáncer (a excepción del tabaco), cuesta ponerlos de manifiesto y su estudio requiere un gran tamaño muestral y un seguimiento muy prolongado. Se decidió no incluir los antecedentes personales en los análisis estadísticos finales por saturar los modelos multivariables desde el inicio e impedir cualquier otra visión. Su efecto sobre el riesgo subsiguiente es muy conocido y en este estudio, previa advertencia, hemos primado el enfoque etiológico sobre otros factores de menor presencia estadísti- ca pero de interés clínico y epidemiológico superior como la dieta u otros aspectos clínicos o antropométricos. Cuando los análisis se han realizado por grandes causas específicas, nuestros datos ratifican la asociación entre tabaco y cáncer, pero no entre tabaco y enfermedades cardiovasculares. La mortalidad total y cardiovascular comparten cuatro factores de riesgo cardiovascular conocidos, edad, sexo y de manera muy destacada por la elevada magnitud de la asociación, creatinina y diabetes. La diabetes es un potente factor de riesgo cardiovascular conocido o una enfermedad cardiovascular en sí misma tal como los datos muestran. La creatinina creemos que fundamentalmente expresa el daño renal presente en la diabetes o hipertensión arterial avanzada y ha sido considerada factor de riesgo cardiovascular per se ${ }^{19,20}$.

Mención especial merece la GGT. Se encuentra en el estudio por ser un parámetro habitualmente incluido en el analizador bioquímico automático. En el momento del diseño del estudio DRECE se desconocía su efecto de riesgo asociado a la mortalidad total y por otras causas. Su presencia se debe a la cirrosis y afectación hepática en el cáncer, sin que parezca estar en relación a la mortalidad cardiovascular subyacente. El elevado número de personas diabéticas entre los fallecidos y la reciente vinculación en varios estudios de la GGT al síndrome metabólico, y la asociación de este último a la mortalidad cardiovascular ${ }^{21,22}$ podrían habernos hecho pensar en ese sentido pero los datos no lo confirman.

El Índice de Masa Corporal (IMC) y la dieta son los grandes ausentes del perfil de riesgo. Ningún dato de la dieta se asoció a la mortalidad total, cardiovascular, o por cáncer. La información sobre la dieta se obtuvo por un cuestionario semicuantitativo de hábitos alimentarios. Quizás aquí también los fallecimientos recopilados hasta la fecha resulten insuficientes para poner de manifiesto relaciones con nutrientes $\mathrm{o}$ 
patrones alimentarios específicos. Es cierto que (datos no mostrados) la dieta de la cohorte DRECE permanece muy estable a lo largo del tiempo con distribución relativamente homogénea de los perfiles lipídicos. En definitiva se obtuvo un patrón de mortalidad en el que predominó el cáncer como causa más frecuente. Aparecieron factores de riesgo clásicos asociados a las principales causas pero con una magnitud muy superior a la tradicional en el caso de la diabetes y creatinina. La diabetes mellitus emerge como un factor muy llamativo asociado a mortalidad precoz. La ratio de mortalidad de cáncer/cardiovascular es de 1,2 y podría ser un fenómeno extensible al conjunto de la población en breve plazo.

\section{BIBLIOGRAFÍA}

1. Gómez-Gerique JA, Gutierrez-Fuentes JA, Montoya MT, Porres A, Rueda A, Avellaneda A et al. Perfil lipídico de la población española: Estudio DRECE (dieta y riesgo de enfermedad cardiovascular en España). Grupo de estudio DRECE. Med. Clin (Barc.). 1999; 113: 730-735.

2. Ballesteros-Pomar MD, Rubio-Herrera MA, Gutiérrez-Fuentes JA, Gomez-Gerique JA, Gómez-De-La-Cámara A et al. Dietary habits and cardiovascular risk in the Spanish population: the DRECE study (I). Ann. Nutr. Metab. 2000; 44: 108-114.

3. Ministerio de Sanidad y Consumo. Estudio DRECE. Dieta y Riesgo Cardiovascular en España. Madrid: Ministerio de Sanidad y Consumo; 1993.

Anexo I

\title{
Grupo DRECE-III
}

\begin{abstract}
E Juncadella i García (Centro de Salud A B C de Hospitalet); E Melús Palazón, MJ Morales Gregorio, P Pitarque Cargallo, E Mayayo Castillejo, I González Gómez de Segura, MI Sancho Giner, AM Aznárez García, F Ibañez García, ME Marco GayaHRe, P Sebastian Villán, E Muñoz Novella, MA Montañés Gracia, S Murciano González, V Peg Rodríguez (Centro de Salud Actur-Sur); R Saénz Guallar, A Abos Zueco, J Pastor Espinosa, JJ Berlanga Rubio, A García García, ME Estopiñán Estupiñán, B Altaba Sanz, I Castellano Juste, Carmen Burgues Valero (Centro de Salud de Alcañiz) A Aguiar Bautista, V del Rosario Sánchez, MC Gómez Medina, M Martel López, D Ruano López (Centro de Salud de Agüimes); A García BaHRientos,E Hernández Hernández,M Badia Savidó,S Puerto Balete (Centro de Salud Bañeres); J Boned Izued, J Codes Gómez, M C Ortega Calleja, F Sancho Durán (Centro de Salud Calatayud Sur); R Provencio Hernando, FJ Peiró Cifuentes (Centro de Salud de Budia-Guadalajara) M A Diéz García, M Cáceres Hernández (C S Casa del Barco); J B Gómez Castaño, F Fernández (Centro de Salud Cieza-Murcia); E Peréz Calzada, MV Alonso Pérez de Ágreda, M Y Del Campo Ciruelos, M T Díaz Benito, M A González Ramos, C González Ramos (Centro de Salud Federico García Lorca); C Sánchez Arce, T Casaseca Calvo (Centro de Salud General Ricardos); O Pascual Gil (Centro de Salud Guadalajara Sur); C Lasa Unzúe, JD García Díaz (Hospital Universitario Príncipe de Asturias, Alcalá de Henares); F Almagro Múgica, E Yetano LaHRazabal, E Intxaurza Hernández (Centro de Salud Lasarte-Hospital Guipúzcua); IM Socias Buades, M Campo Vázquez, M Garau Miquel, F Ramón Roselló, MJ Barea Mestre, M Barceló Morey (Centro de Salud de Manacor); F López SimaHRo, S Miravet Jimenez, J Fortea López, I Verges Macario (C S de Martorell); M I del Cura González, C Reverte Asuero (Centro de Salud Mendiguchia -CaHRiche); J I Sedano García, E Angulo Vallejo, M A Martínez Solórzano, M Santos Lago ( Centro de Salud Miranda- Este); J Isasia Ballestero, M J Martín Martín, M Monasterio Bazán, C Alonso Canosa, Y ZoHRaquino Muñoz, L E Gómez Rodriguez (Centro de Salud Pobo de Dueñas-Guadalajara); F San Juan García, A Cruz Macías, S Vilariño Román (Centro de Salud Plaza de Argel); Adalberto SeHRano Cumplido, Javier Antón Ortega, Javier del Río Fernández, Lourdes Uribe-EtxebaHRía García, Natividad López Miguel, Emilio Borobio del Campo, Julia Marín Vieites, Adelina García García (Centro de Salud de Portugalete- Osakidetza); M J Castellanos Alonso, M Pellitero Espina (Centro de Salud Reina Sofía); H Cardona Castellano, M D CaHRascosa FeHRera, J MaHRero Brito (Centro de Salud San Gregorio- Telde); L M Fontenla Devesa, M C Luna BaHRós, M C Paz Silva, R Rubianes Soto, M D Durán Pereira, R D Martínez Meijide, N Silva García (Centro de Salud de San Roque-Villagarcía de Arosa); M C Velicia Peña, M Domínguez Sardiña, J Mosquera Nogueira, M Rodríguez Ríos, V J Diéguez Pereira, C Gabián Pereira, M Velhas Pereira, X M Parente Mojón, J A Río Orgueira, C Cruces Artero, M A Rionegro López(Centro de Salud Sardoma) P González Aído, S Baleato González, A Álvarez Caride (Centro de Salud Vite) C Lamas, Subdirección de (Atención Sanitaria SAS) P FeHRando P, D Lora, DE la Cruz J, Cancelas P, Magán P (Unidad de Investigación Hospital Universitario 12 de Octubre).
\end{abstract}


4. Kromhout D. On the waves of the Seven Countries Study. A public perspective on cholesterol. Eur. Heart J. 1999; 20: 796-802.

5. Menotti A, Puddu PE, Lanti M, Kromhout D, Blackburn H, Nissinen A.. Twenty-five-year coronary mortality trends in the seven countries study using the accelerated failure time model. Eur. J. Epidem. 2003; 18: 113-22.

6. Tomás-Abadal L, Varas-Lorenzo C, BernadesBernat E, Balaguer-Vintró I. Coronary risk factors and a 20 year incidence of coronary heart disease and mortality in a Mediterranean industrial population. The Manresa study, Spain. Eur. Heart J. 1994; 15: 1028-36.

7. Dawber TR. The Framingham study. The epidemiology of atherosclerotic disease. Cambridge. Harvard University Press; 1980.

8. Ballesteros-Pomar MD, Rubio-Herrera MA, Gutiérrez-Fuentes JA, Gómez-Gerique JA et al. Dietary Habits and Cardiovascular Risk in the Spanish Population: The DRECE Study (II) Micronutrient Intake. Ann. Nutr. Metab. 2000; 44: 177-182.

9. Gutiérrez Fuentes JA, Gomez-Jerique J, Gomez de la Cámara A, 1 Rubio MA, García Hernández A, Aristegui I. Dieta y riesgo cardiovascular en España (DRECE II). Descripción de la evolución del perfil cardiovascular. Med. Clin. (Barc.). 2000; 115: 726-729.

10. Vioque $\mathbf{J}$ and Gonzalez L. Validity of a food frequency questionnaire (preliminary results). Eur. J. Clin. Nutri. 1991; 1: 19-20.

11. Müller-Nordhon J, Binting S, Roll S, and Willich $\mathrm{SN}$. An update on regional variation in cardiovascular mortality within Europe. Eur. Heart J. 2008; 29:1316-1326.

12. Instituto Nacional de Estadística. Demografía y población. Movimiento natural de la población. Disponible : http://www.ine.es/inebase/cgi/

13. Ministerio-de-Sanidad-y-Consumo. Mortalidad por causa de muerte 1981-2004. España y comunidades autónomas. Madrid: Ministerio de Sanidad y
Consumo; Disponible:http://www.msc.es/estadEstudios/estadisticas/estadisticas/estMinisterio/mortalidad/home.htm

14. Boix Martínez R, Aragonés Sanz N, Medrano Albero MJ. Tendencias en la mortalidad por cardiopatía isquémica en 50 provincias españolas. Rev. Esp. Cardiol. 2003; 56: 850-856.

15. Villar Álvarez F, Banegas Banegas JR, Donado Campos JM. Las enfermedades cardiovasculares y sus factores de riesgo en España: hechos y cifras. Sociedad Española de Arteriosclerosis. Madrid: Ergon; 2003.

16. Trichopoulou A, Costacou T, Barmia CH, And Tricopoulos D. Adherence to a Mediterranean Diet and Survival in a Greek Population. N. Engl. J. Med. 2003; 348: 2599-2608.

17. Ahmedin Jemal et al. Cancer Statistics, 2005. American Cancer Society CA. Cancer J. Clin. 2005; 55: 10-30.

18. Knoops KT, De Groot LC, Kromhout D et al. Mediterranean diet, lifestyle factors and 10-year mortality in elderly European men and women. JAMA. 2004; 292: 1433-9.

19. Gerstein HC, Mann JF, Yi Q, Ziman B, Dinneen SF, Hoogwerf B. et al. Albuminuria and risk of cardiovascular events, death, and heart failure in diabetic and non-diabetic individuals. JAMA. 2001; 286: 421-6.

20. Wang TJ, Evans JC, Meigs JB, Rifai, Fox CS, D'Agostino RB et al. Low-grade albuminuria and the risks of hypertension and blood pressure progression. Circulation 2005; 111: 1370-6.

21. Guize L, Thomas F, Pannier B, Bean K, Danchin N, Benetos A. Metabolic syndrome: prevalence, risk factors and mortality in a French population of 62000 subjects Bull. Acad. Natl. Med. 2006; 190: 685-97.

22. Lee DS, Evans JC, Robins SJ, Wilson PW, Albano I, Fox CS et al. Gamma glutamyl transferase and metabolic syndrome, cardiovascular disease, and mortality risk: the Framingham Heart Study. Arterioscler. Thromb. Vasc. Biol. 2007; 27: 127-33. 